\title{
Design and Properties of Octaslide Redundant Parallel Kinematics
}

\begin{abstract}
V. Bauma, M. Valášek, Z. Šika
This paper describes the conceptual design process of OCTASLIDE redundant parallel kinematics for a machine tool. Redundantly actuated parallel kinematics is a recently developed new concept for machine tools. It enables all mechanical properties of machine tools to be improved several times simultaneously. This is particularly demonstrated on the design of the OCTASLIDE. This is a concept of a five-axis machine tool centre. The paper describes the critical initial design phases and the accessible mechanical properties. The design process has follow the newly developed design methodology for parallel kinematics machines.
\end{abstract}

Keywords: parallel kinematics, redundant actuation, five axes, machine tool, dexterity, stiffness.

\section{Introduction}

This paper describes the conceptual design process of OCTASLIDE redundant parallel kinematics for a machine tool. Redundantly actuated parallel kinematics is a recently developed new concept for machine tools. It enables all mechanical properties of machine tools to be improved several times simultaneously. This is particularly demonstrated on the design of the OCTASLIDE. This is a concept of five-axis machine tool center. The paper describes the critical initial design phases and the accessible mechanical properties. The design process follows the newly developed design methodology for parallel kinematics machines.

\section{Concept of redundant actuation for parallel kinematics}

Parallel kinematics means kinematic refers to where a body carrying a tool (called a platform) is supported by several independent links (legs) from the frame. Parallel kinematics has the advantage that in principle all drives can be on the frame (decreased moving masses) and that its structure is a truss (increased stiffness). However, it also has severe disadvantages, mainly the occurrence of singular positions in the workspace and a generally smaller workspace due to link collisions.

These problems of parallel kinematics can be removed by the principle of redundant actuation [1]. If the platform of parallel kinematics is supported on a redundant number of legs (links), i.e. on more than the needed number of DOFs -

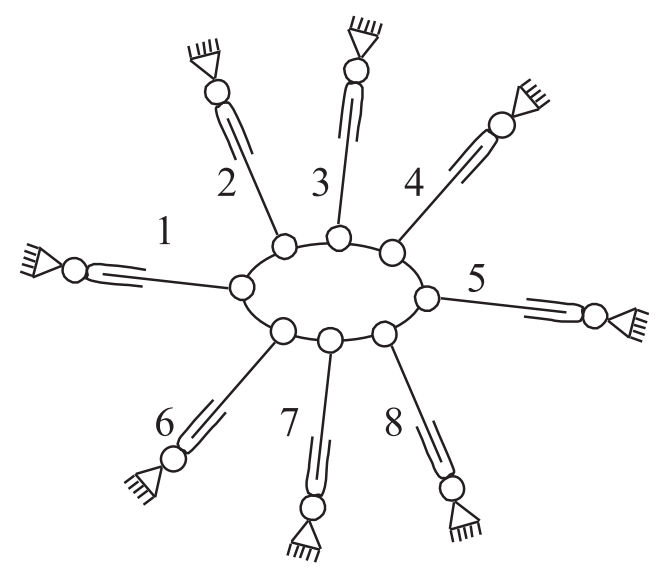

Fig. 1: The concept of redundant actuation of parallel kinematics

in plane, on more than 3 legs, and in space, on more than 6 legs, then the following simplified consideration can be applied. If a certain combination of 6 legs from the redundant number of legs in the given position of the kinematics leads to a singular position, then the other combination of another 6 legs in the same position will be in a non-singular position (Fig. 1). Certainly switching between different selected combinations of legs is just an ideal consideration. The kinematics must use all redundant legs simultaneously and the control must be correspondingly smooth. The actuators of parallel structures can be realized by different principles (Fig. 2), not only by telescopic links.
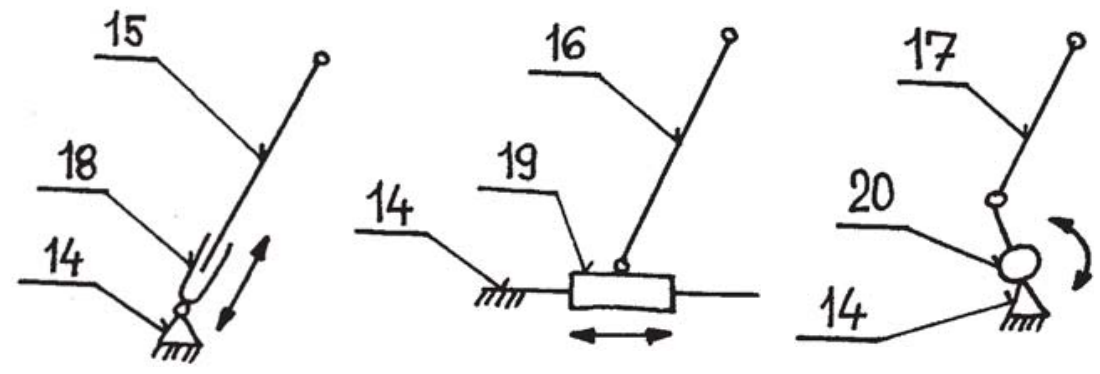

Fig. 2: The different constructions of link actuators (telescopic, sliding, rotational) 
Using telescopic links, the octapod has been proposed as a redundant variant of the traditional hexapod. This paper deals with the conceptual design of redundant parallel kinematics with sliding links.

\section{Design methodology for parallel kinematics}

The design of parallel kinematics is a difficult problem. The fundamental problem is that almost all design parameters are mutually dependent. This leads to significantly increased computational complexity. A special design methodology has therefore been developed for parallel kinematics [2]. This methodology has succeeded in decreasing the computational complexity of the design by decomposing the design process into several hierarchical levels and by using of computational tools capable of computing the mechanical properties globally (for the whole workspace, rather than for one position in it).

\section{Octaslide concept}

The octaslide is a redundant version of the hexaslide. It is a parallel kinematics the links of which have sliding actuators. The principal concept of such a structure is shown in Fig. 3. The platform is suspended on 8 links with actuators, unlike the hexaslide or pentaslide, which are suspended on only 6 or 5 links.

\section{Workspace and dexterity optimization}

The goal is to design the octaslide as a machine tool for a cylindrical workspace with a diameter of $1200 \mathrm{~mm}$ (length is almost unlimited). The platform is again a cylinder with diameter $400 \mathrm{~mm}$ and a length of $700 \mathrm{~mm}$ for axial suspension of the spindle. The goal is to maximize the orientation angles for five-axis machining.

The investigation and optimization were provided on three testing trajectories (Fig. 4). The first is a planar circular trajectory with chords of radius $540 \mathrm{~mm}$ in plane $x-z$, the second is a conical trajectory from the origin with variant deflection angle $\delta$, and the third is a conical trajectory on a circular path in plane $x-z$ of radius $540 \mathrm{~mm}$ with variant deflection.

First, the hexaslide was optimized. Two structures were investigated. The first kinematic structure is symmetric (Fig. 5). The second structure is asymmetric, with links of different lengths (Fig. 6).

The influence of structure asymmetry on the important kinematic property of dexterity (characterizing the distance from the singular position) is very strong. A comparison of the dexterity behaviour of the two hexaslide structures on three testing trajectories is shown in Fig. 9 (left).

The same investigation was done for the octaslide. Two structures were designed and optimized - symmetric and

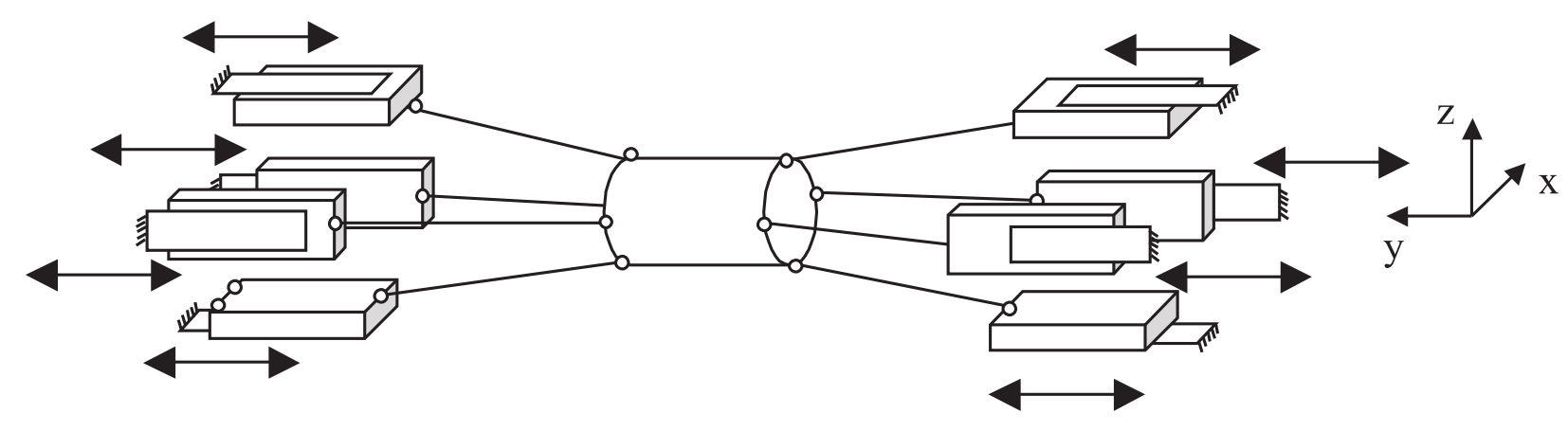

Fig. 3: Kinematic concept of the octaslide
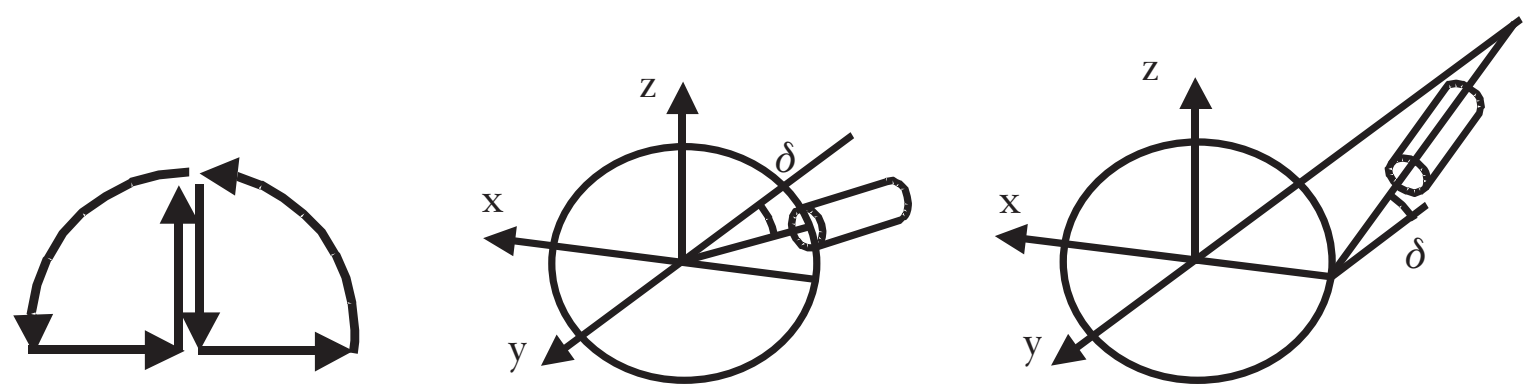

Fig. 4: Three testing trajectories 

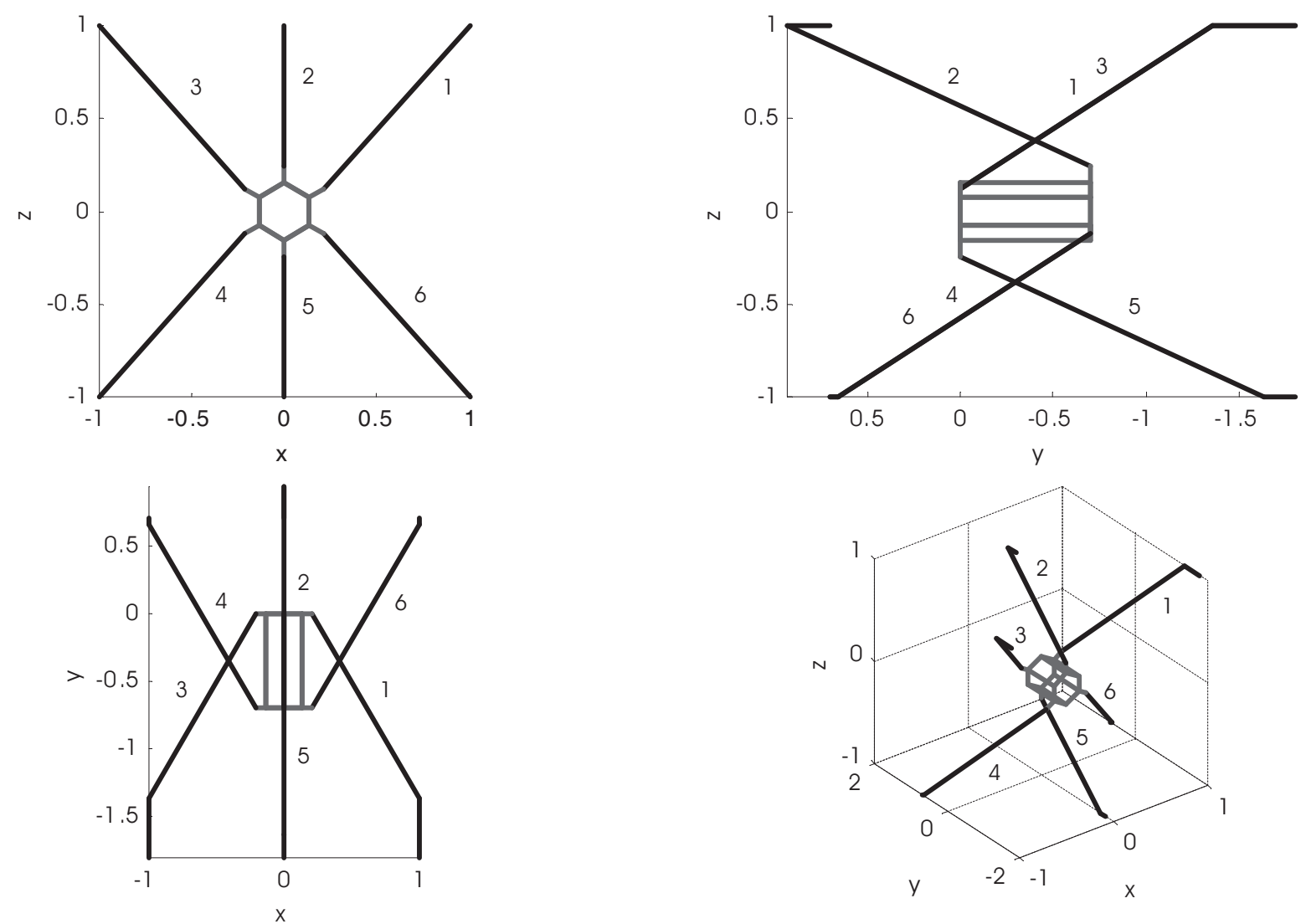

Fig. 5: Hexaslide with a symmetric structure
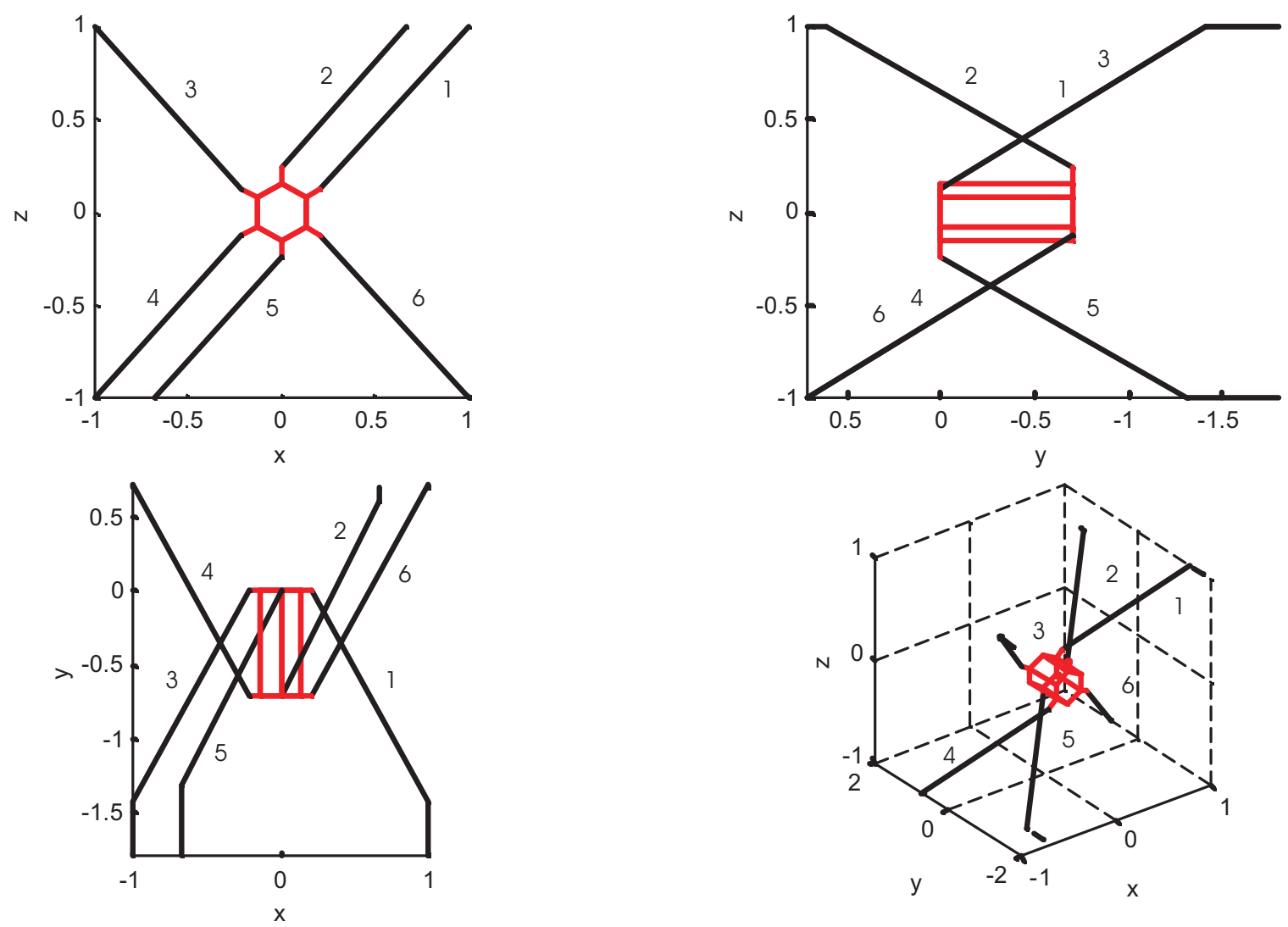

Fig. 6: Hexaslide with an asymmetric structure (different lengths of links) 

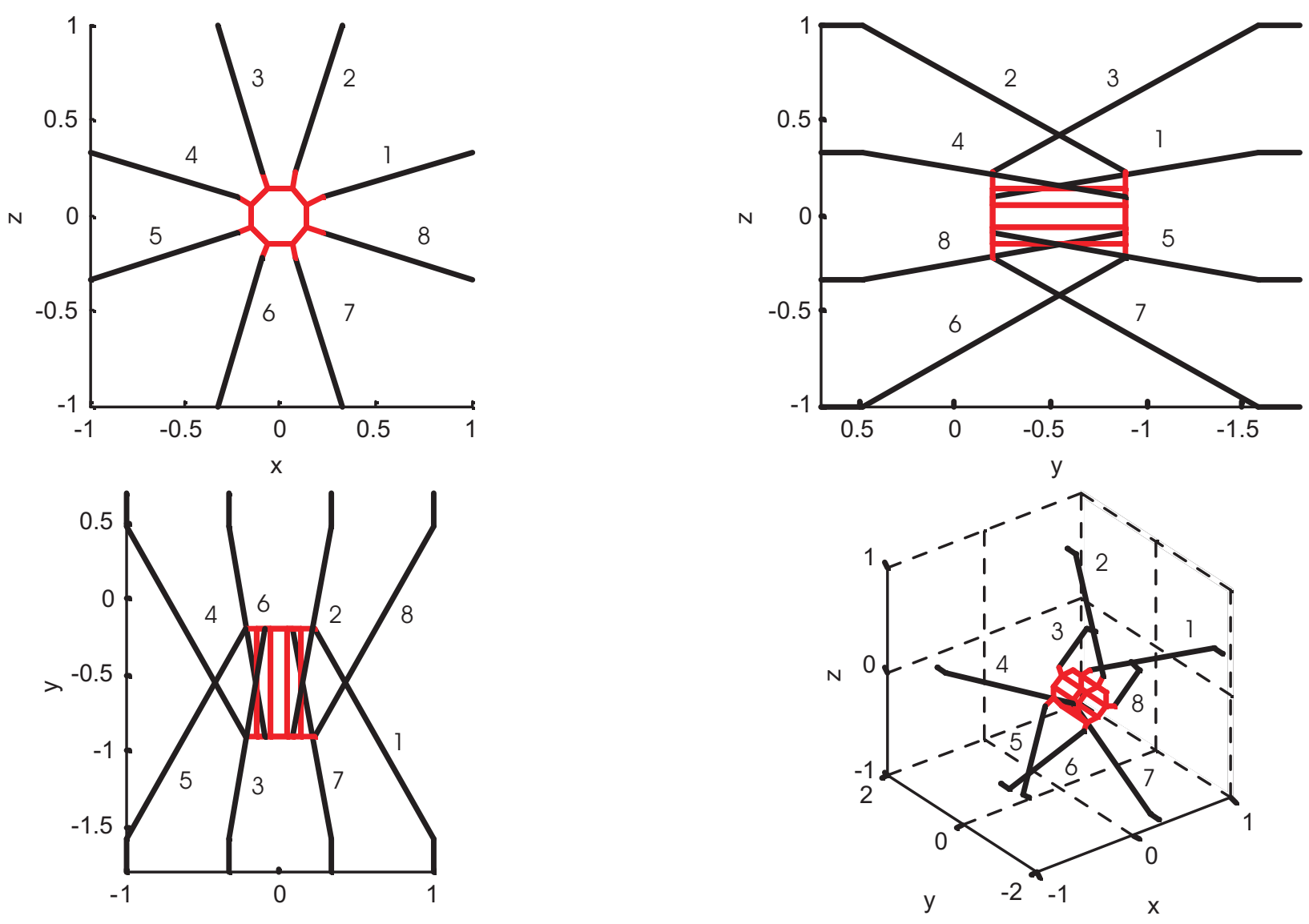

Fig. 7: Octaslide with a symmetric structure
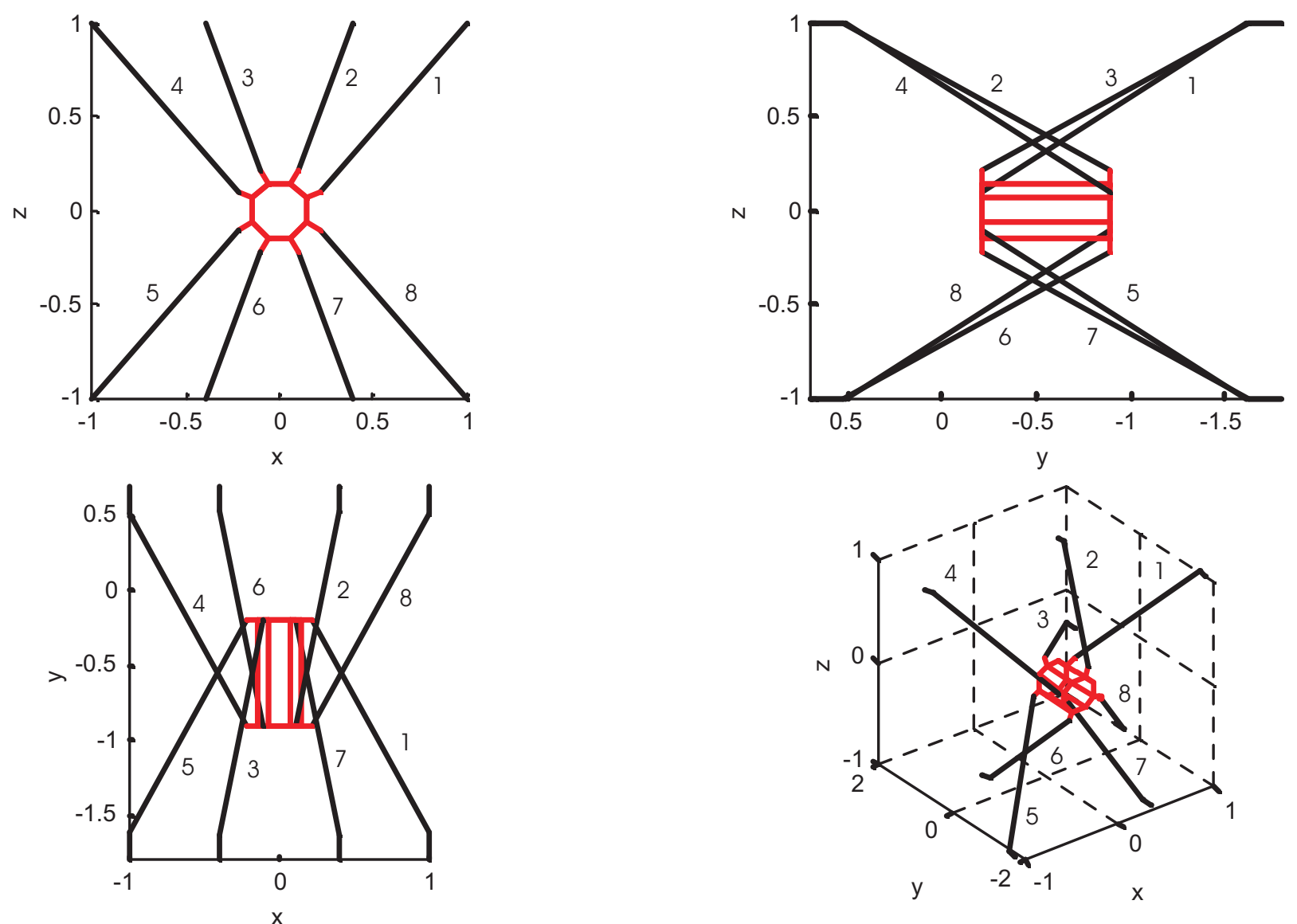

Fig. 8: Octaslide with an asymmetric structure (different lengths of links) 

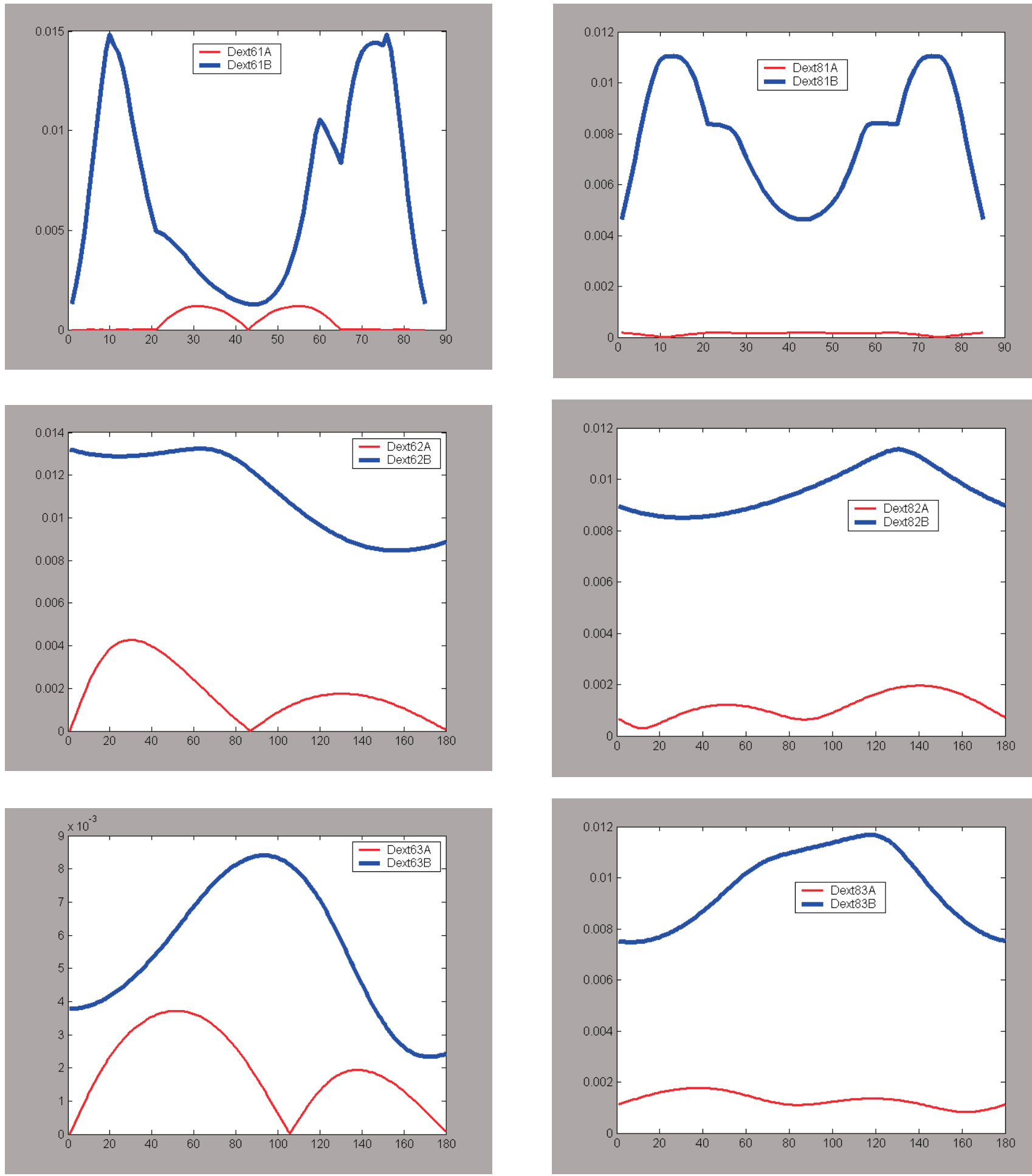

Fig. 9: Dexterity on three testing trajectories for the hexaslide (left) and the octaslide (right)

asymmetric (Figs. 7 and 8). The influence of these structures on dexterity on the testing trajectories is again very strong. A comparison of the dexterity behaviour of the two octaslide structures on three testing trajectories is shown in Fig. 9 (right).

A comparison of the dexterity on testing trajectories of the optimized hexaslide and octaslide is shown in Fig. 10 a, b, c. The behaviour of the octaslide is significantly better as the dexterity minimum is increased and its variation is decreased.
The principle of asymmetry was further investigated and the structure of the octaslide was optimized (Fig. 11) by varying the link lengths and their positioning on the frame. The dexterity was further significantly improved above the previous levels (Fig. 10c) comparing the optimized hexaslide and the two octaslides. The octaslide from Fig. 11 has better dexterity in almost the whole workspace.

Simultaneously with the dexterity, the declination angles characterizing the orientation capabilities were also investi- 


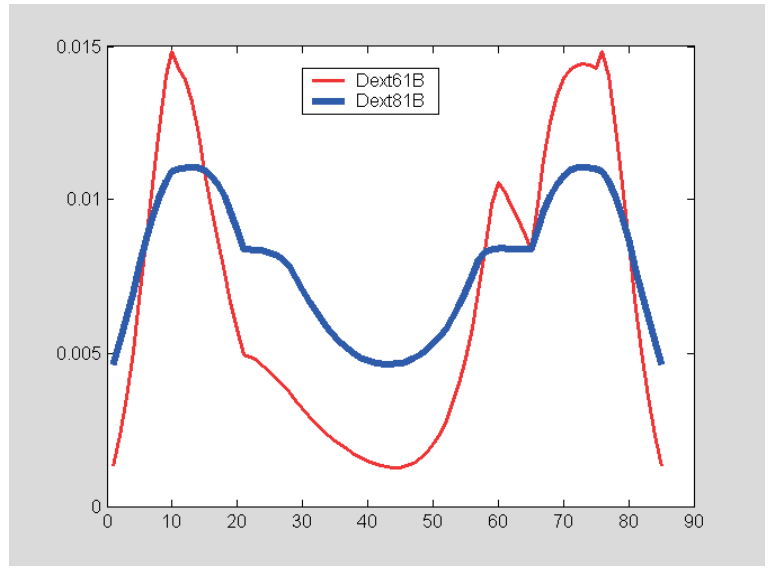

a)

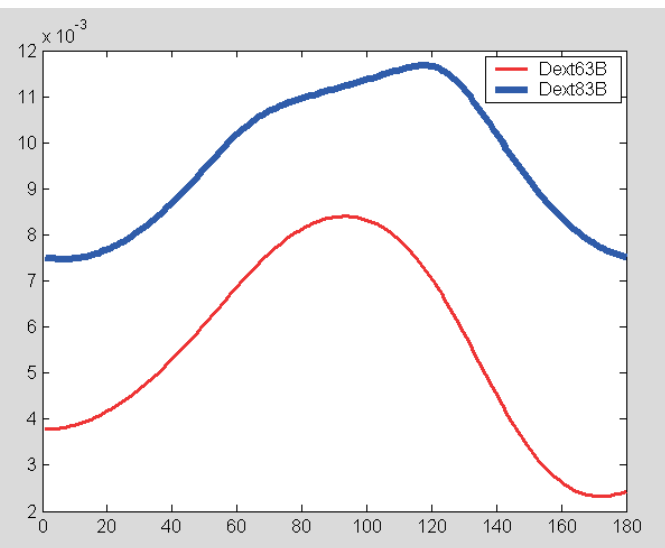

c)

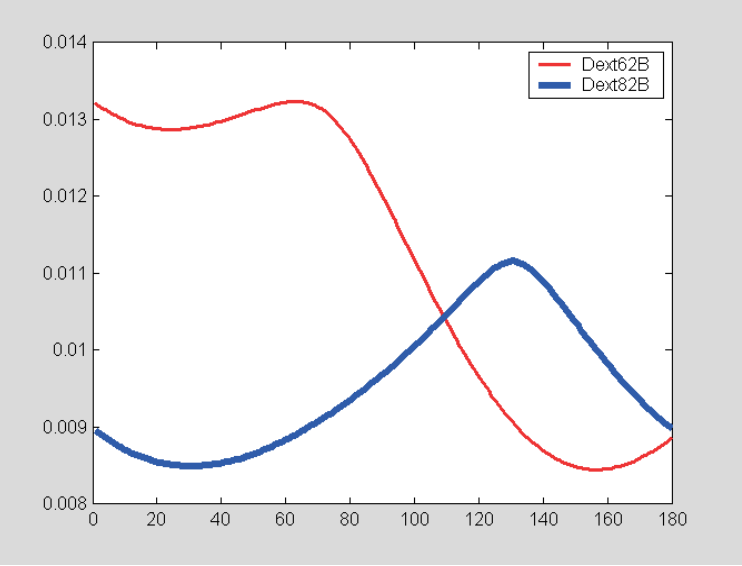

b)

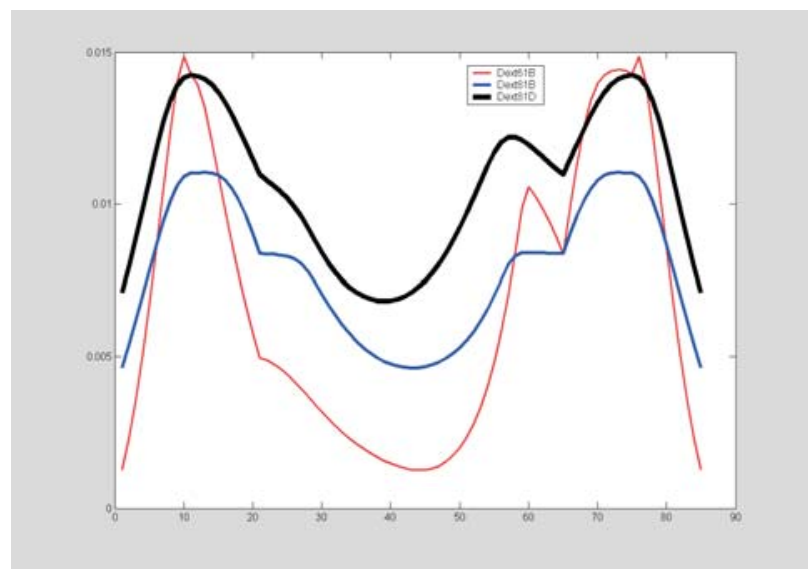

d)

Fig. 10: Dexterity comparison on three testing trajectories of optimized hexaslide and octaslide

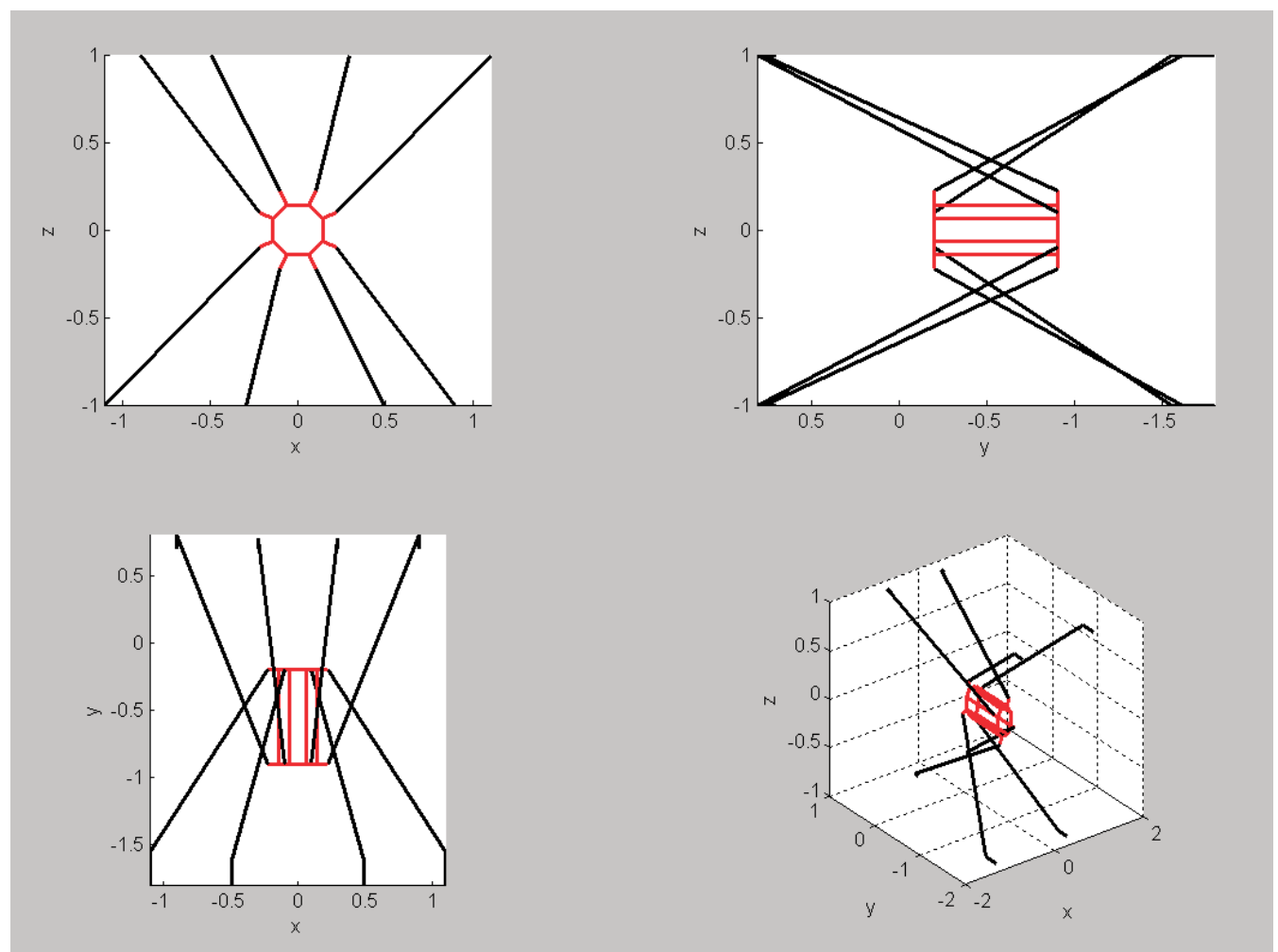

Fig. 11: Further optimized octaslide with increased asymmetry of links 

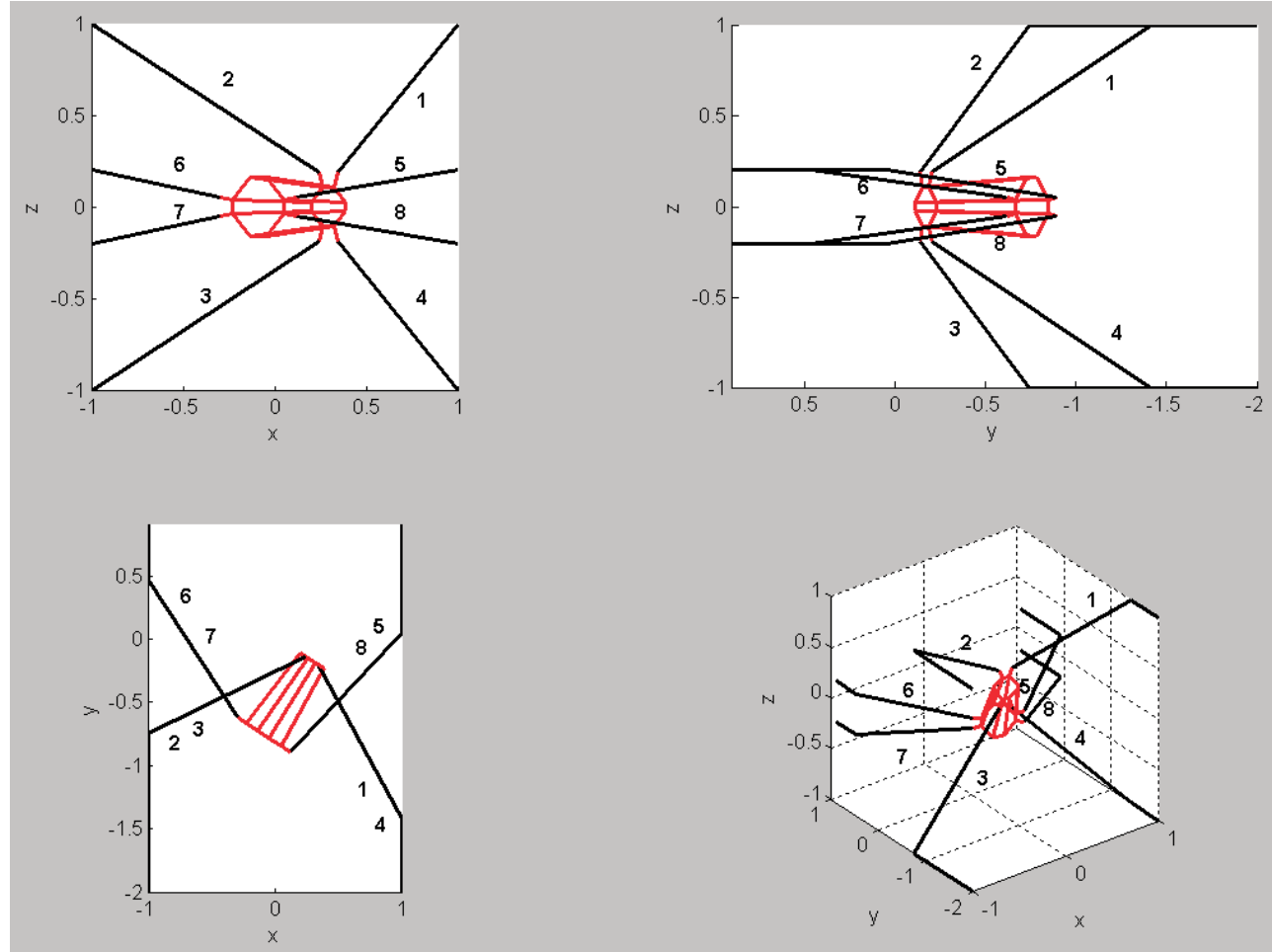

Fig. 12: Octaslide with a conical platform
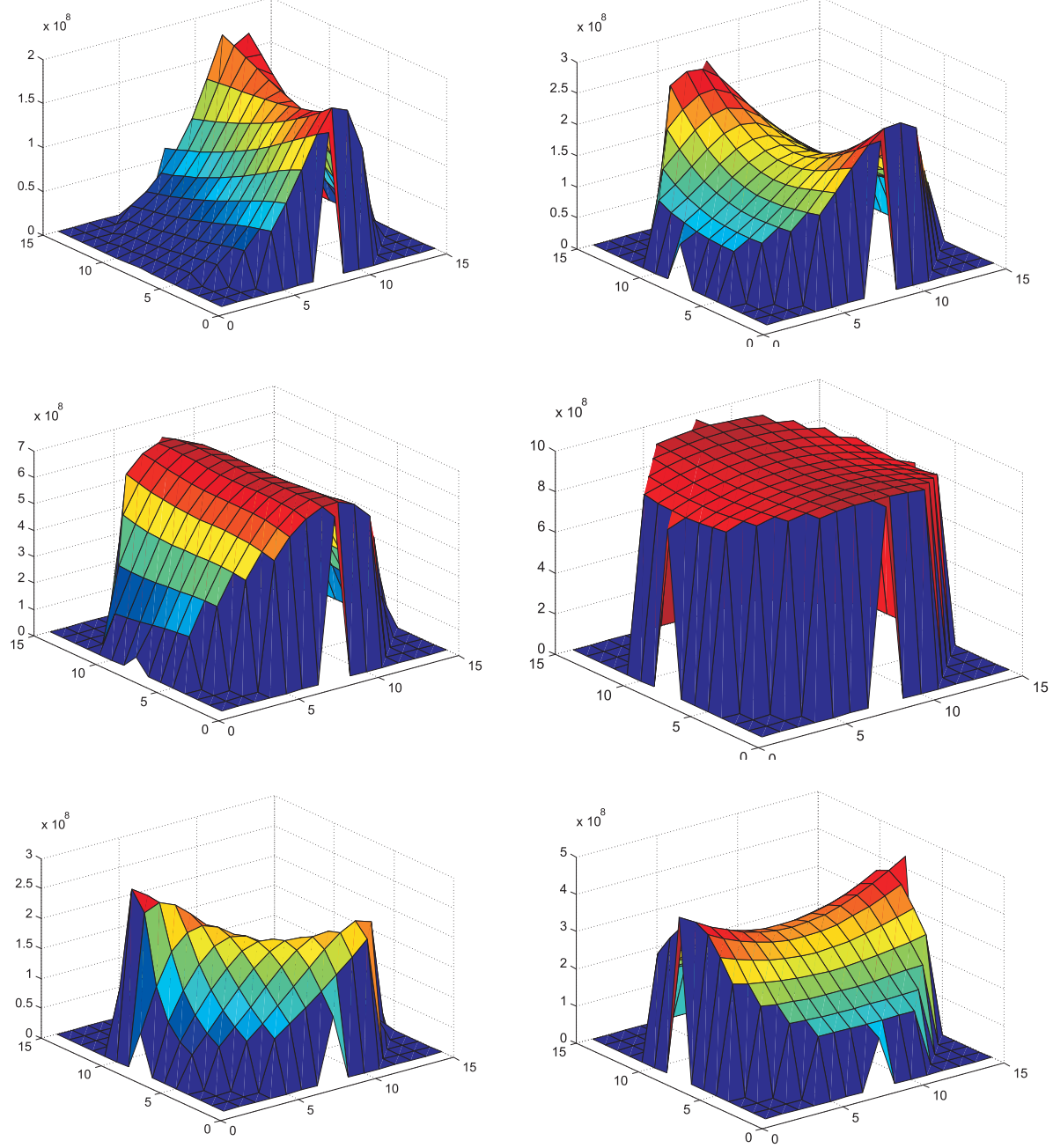

Fig. 13: Stiffness in the workspace for the hexaslide (left) and the octaslide (right) in directions x, y, z 
gated and optimized. The declination of the hexaslide for trajectory 2 is $14^{\circ}$, and for trajectory 3 it is $13^{\circ}$. The declination of the octaslide for trajectory 2 is $16^{\circ}$, and for trajectory 3 it is $12^{\circ}$. This means that the two structures are comparable in terms of their orientation capabilities. Then the structure of the octaslide was further optimized. The platform was conical instead of cylindrical (Fig. 12). This modification enables the declination angle to be extended to $33^{\circ}$.

\section{Octaslide stiffness}

The stiffness in the whole workspace was compared for the two optimized variants of the hexaslide and the octaslide. They are shown in Fig. 13. The comparison of maximum, minimum and average values demonstrates the significant superiority of the octaslide. The increase in the maximum values is $65-74 \%$, and the increase in the average values is $43-54 \%$.

\section{Conclusions}

The concept of redundant actuation enables the design of new parallel kinematics with significantly improved mechanical properties. This has again been confirmed by the design of the octaslide.

\section{Acknowledgment}

The authors appreciate the support of MSMT project J04/98:212200008.

\section{References}

[1] Valášek M. et al: "New Concept of Redundant Parallel Robot". In: Proc. of Mechatronics and Robotics 97, VUT Brno, Brno 1997, p. 269-274.

[2] Valášek M., Šika Z., Bauma V., Vampola T.: Design Methodology for Redundant Parallel Robots, in Proc. of AED 2001, 2nd Int. Conf. on Advanced Engineering Design, Glasgow, 2001, p. 243-248.

Ing. Václav Bauma, CSc.

phone: +420224357373

e-mail:vaclav.bauma@fs.cvut.cz

Prof. Ing. Michael Valášek, DrSc.

phone: +420224357361

fax: +420224916709

e-mail: michael.valasek@fs.cvut.cz

Ing. Zbyněk Šika, Ph.D.

phone: +420 224357452

e-mail: zbynek.sika@fs.cvut.cz

Department of Mechanics

Czech Technical University in Prague

Faculty of Mechanical Engineering

Karlovo nám. 13

12135 Praha 2, Czech Republic 